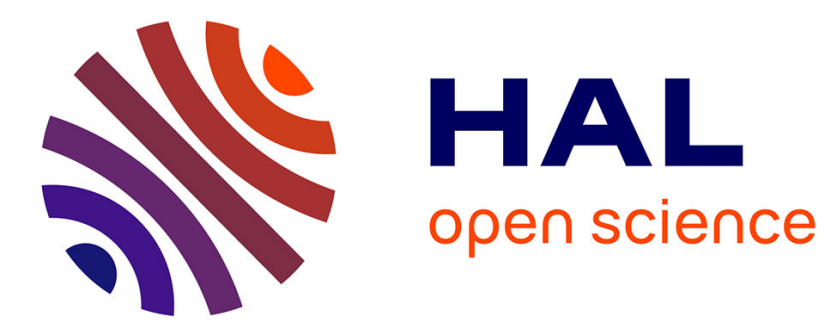

\title{
Tests for Trend: a Simulation Study
}

Abdullah Almasri

\section{To cite this version:}

Abdullah Almasri. Tests for Trend: a Simulation Study. Communications in Statistics - Simulation and Computation, 2010, 39 (03), pp.598-611. 10.1080/03610910903528327 . hal-00567042

\section{HAL Id: hal-00567042 \\ https://hal.science/hal-00567042}

Submitted on 18 Feb 2011

HAL is a multi-disciplinary open access archive for the deposit and dissemination of scientific research documents, whether they are published or not. The documents may come from teaching and research institutions in France or abroad, or from public or private research centers.
L'archive ouverte pluridisciplinaire HAL, est destinée au dépôt et à la diffusion de documents scientifiques de niveau recherche, publiés ou non, émanant des établissements d'enseignement et de recherche français ou étrangers, des laboratoires publics ou privés. 


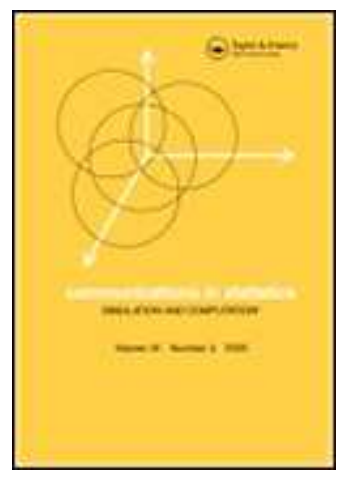

\section{Tests for Trend: a Simulation Study}

\begin{tabular}{|c|c|}
\hline Journal: & Communications in Statistics - Simulation and Computation \\
\hline Manuscript ID: & LSSP-2009-0190.R1 \\
\hline Manuscript Type: & Original Paper \\
\hline $\begin{array}{r}\text { Date Submitted by the } \\
\text { Author: }\end{array}$ & 23-Oct-2009 \\
\hline Complete List of Authors: & $\begin{array}{l}\text { Almasri, Abdullah; Växjö University, Department of Economics and } \\
\text { Statistics }\end{array}$ \\
\hline Keywords: & Discrete Wavelet Transform, Periodogram, Long Memory Process \\
\hline Abstract: & $\begin{array}{l}\text { In this study we use the wavelet analysis to construct a test } \\
\text { statistic } \\
\text { to test for the existence of a trend in the series. We also propose a } \\
\text { new } \\
\text { approach for testing the presence of trend based on the } \\
\text { periodogram of } \\
\text { the data. Since we are also interested in the presence of a long- } \\
\text { memory } \\
\text { process among the data, we study the properties of our test } \\
\text { statistics } \\
\text { under different degrees of dependency. We compare the results } \\
\text { when } \\
\text { using the band periodogram test and the wavelet test with results } \\
\text { obtained by applying the ordinary least squares (OLS) method } \\
\text { under } \\
\text { the same conditions. }\end{array}$ \\
\hline \multicolumn{2}{|c|}{$\begin{array}{l}\text { Note: The following files were submitted by the author for peer review, but cannot be converted } \\
\text { to PDF. You must view these files (e.g. movies) online. }\end{array}$} \\
\hline TestforTrend.zip & \\
\hline
\end{tabular}




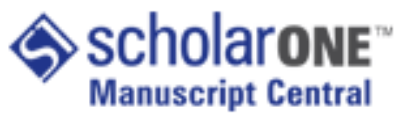




\title{
Tests for Trend: a Simulation Study
}

\author{
Abdullah Almasri \\ Department of Economics and Statistics Växjö University, Sweden
}

\begin{abstract}
In this study we use the wavelet analysis to construct a test statistic to test for the existence of a trend in the series. We also propose a new approach for testing the presence of trend based on the periodogram of the data. Since we are also interested in the presence of a long-memory process among the data, we study the properties of our test statistics under different degrees of dependency. We compare the results when using the band periodogram test and the wavelet test with results obtained by applying the ordinary least squares (OLS) method under the same conditions.
\end{abstract}

\section{Introduction}

A common issue in time series analysis is the decomposition of the different components of variation. In some applications, it is important to decompose a time series into a low frequency (trend) component, and a high frequency (noise and periodicity) component. The multiresolution analysis is a convenient setting for decomposing and describing the different scales of variation in the data, and the periodogram is a simple way for decomposing the variation into low and high frequencies. It seems natural to turn the attention to other tests which are based on the low frequency part; in wavelet domain as well as in frequency domain. In this study we use the wavelet analysis to construct a test statistic to test for the existence of a trend in the series. We also propose a new approach for testing the presence of trend based on the periodogram of the data. Since we are also interested in the presence of a long-memory process among the data, we study the properties of our test statistics under different degrees of dependency. We compare the results when using the band periodogram test and the wavelet test with results obtained by applying the ordinary least squares (OLS) method under the same conditions. 
A major problem in practice is the discrimination of an $\operatorname{FD}(d)$ process with trend from an $\mathrm{FD}(d)$ process without trend, since $\mathrm{FD}(d)$ processes for $0<d<\frac{1}{2}$ usually look like having some trends. This makes it hard to distinguish between these processes. Several authors have considered this problem. Smith (1993) adapts the OLS estimator to test for trend in temperature time series. Deo and Hurvich (1998) investigate the use of OLS estimators to estimate the linear trend for a time series in the presence of additive LM noise. Künsch (1986) provides a method to discriminate between small monotonic trends and LM processes by examining the distribution of the periodogram. Brillinger (1994) and Brillinger (1996) apply the wavelet transform by using the Haar wavelet to provide pointwise confidence intervals for a trend estimation. Craigmile (2000) and Almasri (2000) use the Daubechies wavelet filters for testing the trend in the presence of the FD processes. In the present paper we develop tests for trend which are based on on the low frequency part; in wavelet domain as well as in frequency domain.

The paper is organised as follows: section 1 gives an introduction. The methodology introduced in section 2 , in section 3 we define the trend and trend tests while in section 4 we present a simulation study, and finally, we give a short summary and conclusions in section 5 .

\section{Methodology}

In this section we will introduced the theory that has been used in this study.

\subsection{Fractional Difference Processes}

The autoregressive fractionally integrated moving average (ARFIMA) processes introduced by Granger and Joyeux (1980) and Hosking (1981) are the most widely used class of long-memory models. A process $\left\{X_{t}\right\}$ follows an $\operatorname{ARFIMA}(p, d, q)$ process if

$$
\Phi(B)(1-B)^{d} X_{t}=\Theta(B) \epsilon_{t}
$$

where $\left\{\epsilon_{t}\right\}$ are identically independent distributed (i.i.d) with zero mean and $\sigma_{\epsilon}^{2}$ variance and where $\Phi(B)=1-\phi_{1} B-\ldots-\phi_{p} B^{p}$ and $\Theta(B)=$ $1-\theta_{1} B-\ldots-\theta_{q} B^{q}$ are polynomials of order $p$ and $q$ in the backshift operator 
$B$, i.e., $B X_{t}=X_{t-1}$, and $d$ is the difference parameter and is allowed to take fractional values. In this work we study the simplest type of $\operatorname{ARFIMA}(p, d, q)$ process, i.e., $\operatorname{ARFIMA}(0, d, 0)$, which is called the Fractional Difference (FD) process

$$
(1-B)^{d} X_{t}=\epsilon_{t}
$$

and denoted $\mathrm{FD}(d)$. When $0<d<\frac{1}{2}$ the process is called a stationary Long-Memory process (LM). The spectrum has a pole at zero frequency, and the process exhibits slowly decaying auto-covariances. When $d=0$ in (1), an FD process becomes a white noise process. When $d \geq \frac{1}{2}$ the process is a non-stationary process. Such process will not be considered further here.

\subsection{The periodogram}

The periodogram was first introduced by Schuster (1898) and was used to search for hidden periodicities and, naturally, it became a tool for testing the presence of periodic components.

Consider a stationary process $\left\{Z_{t}\right\}$ - which we assume has zero mean and absolutely continuous spectral density function (SDF) given by $S(f)-$ with autocovariance sequence (acvs) given by $s_{\tau}, \tau=0, \pm 1, \pm 2, \ldots$ The following relationship between the spectral density and autocovariance sequence holds,

$$
S(f)=\sum_{\tau=-\infty}^{\infty} s_{\tau} e^{-i 2 \pi f \tau} \text { for } f \in\left(-\frac{1}{2}, \frac{1}{2}\right]
$$

This relation holds if the auto-covariance sequence (acvs) $s_{\tau}$ is square summable, i.e., if

$$
\sum_{\tau=-\infty}^{\infty} s_{\tau}^{2}<\infty
$$

For LM processes the acvs is not necessarily a square summable sequence, so that the relation (3) does not always hold.

A naïve estimator of the spectrum is given by the periodogram, in which the autocovariance in (2) is replaced by a biased estimator,

$$
\hat{s}_{\tau}= \begin{cases}\frac{1}{N} \sum_{t=1}^{N-|\tau|} Z_{t} Z_{t+|\tau|}, & \text { for }|\tau| \leq N-1 \\ 0, & \text { for } \tau>N-1 .\end{cases}
$$


The periodogram is hence given by

$$
\hat{S}(f)=\sum_{\tau=-(N-1)}^{N-1} \hat{s}_{\tau} e^{-i 2 \pi f \tau}=\frac{1}{N}\left|\sum_{t=1}^{N} Z_{t} e^{-i 2 \pi f t}\right|^{2}, \quad f \in\left(-\frac{1}{2}, \frac{1}{2}\right] .
$$

The periodogram is often evaluated at the Fourier frequencies $\varphi_{k}=k / N$, $k=-\left\lfloor\frac{N-1}{2}\right\rfloor, \ldots,\left\lfloor\frac{N}{2}\right\rfloor$, where $\lfloor x\rfloor$ is the greatest integer less than or equal to $x$. The periodogram can be a good approximation to the true spectrum in cases with small spectral range, in other cases it can be an extremely poor estimate of the spectrum. The disadvantages of the periodogram are well documented, readers are referred to Priestley (1981, Ch. 6) and Percival and Walden (1993, Ch. 6). A modification that improves the properties of the periodogram is tapering. Tapering is a common technique to reduce the bias of the periodogram. In this paper we will work with the raw periodogram without taking into account the tapering technique. The technique can however be extended in the future by using such improving techniques.

\subsection{The band Periodogram}

In this section we introduce the band periodogram that has been introduced by Almasri(2000). This approach can help us in analysing the FD processes. The main idea behind this approach is to divide the Fourier frequencies into different frequency intervals or bands, matching the band-pass of the DWT, i.e. $\left(2^{-(j+1)}, 2^{-j}\right]$. Each band in this case of the FD process might be regarded as a bounded spectrum of a stationary process. We shall now investigate the properties of the spectrum and its estimate at different bands. Before we study the band spectrum and the band periodogram, we have to define the spectral density function (SDF) and the periodogram.

For a stationary process $\left\{Y_{t}\right\}$ with a $\operatorname{SDF} S(f)$ that is continuous over the Nyquist range, it can be shown that, subject to the finiteness of certain high order moments,

$$
\hat{S}(f) \stackrel{\mathrm{d}}{=} \begin{cases}S(f) \chi_{2}^{2} / 2, & \text { for } 0<f<f_{(N)} \\ S(f) \chi_{1}^{2}, & \text { for } f=0 \text { or } f_{(N)}\end{cases}
$$


asymptotically as $N \rightarrow \infty$. Furthermore, $\hat{S}(f)$ and $\hat{S}\left(f^{\prime}\right)$ are asymptotically independent. We thus have

$$
\begin{aligned}
& \operatorname{var}\{\hat{S}(f)\}= \begin{cases}S(f)^{2}, & \text { for } 0<f<f_{(N)} \\
2 S(f)^{2}, & \text { for } f=0 \text { or } f_{(N)}\end{cases} \\
& \operatorname{cov}\left\{\hat{S}\left(f^{\prime}\right), \hat{S}(f)\right\}=0, \quad 0 \leq f^{\prime}<f<f_{(N)},
\end{aligned}
$$

asymptotically as $N \rightarrow \infty$. Hence, for a $N$ large enough the periodogram ordinates at the grid of Fourier frequencies are all approximately pairwise uncorrelated; i.e.,

$$
\operatorname{cov}\left\{\hat{S}\left(\varphi_{k}\right), \hat{S}\left(\varphi_{l}\right)\right\} \approx 0, \quad k \neq l \quad \text { and } \quad 0 \leq k, l \leq\lfloor N / 2\rfloor
$$

For more details about the spectral analysis, readers are referred to Priestley (1981) and Percival and Walden (1993). Let us now define the band decomposing of the spectrum of the process $\left\{X_{t}\right\}$ with the following spectrum

$$
\begin{gathered}
S(f)=\sum_{j=1}^{J} S_{j}(f) \\
S_{j}(f)= \begin{cases}S(f) I_{\left(2^{-(j+1)}, 2^{-j}\right]}(f) & j=1, \ldots, J-1 \\
S(f) I_{\left[0,2^{-j}\right]}(f) & j=J\end{cases}
\end{gathered}
$$

where $I_{[.]}$is an indicator function, the first part in the right of (9) collectively represents the high-frequency spectrum $S^{H}(f)$ and the second part represents the low-frequency spectrum $S^{L}(f)$.

The basic estimator of $S($.$) is the periodogram and in the same way we define$ the band decomposition of the periodogram

$$
\hat{S}_{j}(f)= \begin{cases}\hat{S}(f) I_{\left[2^{-(j+1), 2-j]}\right.}(f) & j=1, \ldots, J-1 \\ \hat{S}(f) I_{\left[0,2^{-j}\right]}(f) & j=J\end{cases}
$$

We call the first part of (10) the high-frequency band periodogram $\hat{S}^{H}(f)=$ $\sum_{j=1}^{J-1} \hat{S}_{j}(f)$ and the second part the low-frequency band periodogram $\hat{S}^{L}(f)$. 


\subsection{The Discrete Wavelet Transform}

The key idea of the discrete wavelet transform (DWT) is to decompose a series orthogonally into different new series. Let $\mathbf{X}=\left(X_{0}, \ldots, X_{N-1}\right)$ be a data vector of length $N$, where we assume that $N$ is an integer divisible by $2^{J}$, where $J$ is a positive integer. The wavelet and scaling filters are used in parallel to define the DWT i.e., we have two types of coefficients in the DWT based on these two types of filter: the scaling coefficients and the wavelet coefficients. The pyramid algorithm, introduced by Mallat (1989), uses linear filtering operations. The first stage of the pyramid algorithm for computing DWT consists of transforming the time series $\mathbf{X}$ into the $N / 2$ first level scaling coefficients and the $N / 2$ first level wavelet coefficients. There are $J-1$ subsequent stages of the pyramid algorithm. The scaling coefficients for level $j(j=1, \ldots, J)$ are given by

$$
V_{j, k}=\sum_{l=0}^{L-1} g_{l} V_{j-1,2 k+1-l \bmod N_{j-1}} \text { for } \quad k=0, \ldots, N_{j}-1,
$$

and the wavelet coefficients for level $j$ are given by

$$
W_{j, k}=\sum_{l=0}^{L-1} h_{l} V_{j-1,2 k+1-l \bmod N_{j-1}} \text { for } k=0, \ldots, N_{j}-1 \text {, }
$$

where $g_{l}$ is the scaling filter, $h_{l}$ is the wavelet filter, $\mathbf{V}_{0} \equiv \mathbf{X}$ and $N_{j} \equiv N 2^{-j}$. The modulus operator in (11) and (12) is required in order to deal with the boundary of a finite length vector of observations. This operator circularly filters the data, by using a fast filtering algorithm of order $O(N)$. We see from (12) and (11) that at each step we filter the previous level scaling coefficients using either the scaling or wavelet filter, and then subsample the resulting sequence. The DWT can be defined also by matrix calculation. Let $\mathbf{W}_{j} \equiv$ $\left[W_{j, 0}, W_{j, 1}, \ldots, W_{j, N_{j}-1}\right]^{T}, j=1,2, \ldots, J$, and $\mathbf{V}_{J} \equiv\left[V_{J, 0}, V_{J, 1}, \ldots, V_{J, N_{J}-1}\right]^{T}$. The elements of the subvectors $\mathbf{W}_{j}$ correspond to that in (12) and the subvector $\mathbf{V}_{J}$ correspond to that in (11). We then have the analysis equation

$$
\mathbf{W}=\mathcal{W} \mathbf{X}
$$


where $\mathbf{W}$ contains the DWT coefficients, i.e.,

$$
\mathbf{W}=\left(\begin{array}{c}
W_{1} \\
W_{2} \\
\vdots \\
W_{j} \\
\vdots \\
W_{J} \\
V_{J}
\end{array}\right)
$$

and $\mathcal{W}$ is an orthonormal $N \times N$ real-valued matrix whose rows depend on the wavelet filter $h_{l}$, i.e., $\mathcal{W}^{-1}=\mathcal{W}^{T}$, so $\mathcal{W}^{T} \mathcal{W}=\mathcal{W} \mathcal{W}^{T}=\mathbf{I}_{N}$, see Percival and Walden (2000). We can reconstruct our time series from the wavelet coefficients $\mathbf{W}$ by using

$$
\mathbf{X}=\mathcal{W}^{T} \mathbf{W} .
$$

We partition the columns of $\mathcal{W}$ commensurate with the partitioning of $\mathbf{W}$ to obtain

$$
\mathcal{W}^{T}=\left[\mathcal{W}_{1} \mathcal{W}_{2} \ldots \mathcal{W}_{j} \ldots \mathcal{W}_{J} \mathcal{V}_{J}\right]
$$

where $\mathcal{W}_{j}$ is an $N \times N / 2^{j}$ matrix and $\mathcal{V}_{J}$ is an $N \times N / 2^{J}$ matrix. Now, we can define the multiresolution analysis of a series by expressing $\mathcal{W}^{T} \mathbf{W}$ as a sum of several new series, each of which is related to variations in $X$ at a certain scale:

$$
\mathbf{X}=\mathcal{W}^{T} \mathbf{W}=\sum_{j=1}^{J} \mathcal{W}_{j} \mathbf{W}_{j}+\mathcal{V}_{J} \mathbf{V}_{J}=\sum_{j=1}^{J} D_{j}+S_{J}
$$

The terms in (13) constitute a decomposition of $X$ into orthogonal series components $D_{j}$ (detail) and $S_{J}$ (smooth) at different scales and the length of $D_{j}$ and $S_{J}$ coincides with the length of $\mathbf{X}(N \times 1$ vector $)$. Because the terms at different scales represent components of $\mathbf{X}$ at different resolutions the approximation is called multiresolution decomposition. Daubechies (1992) introduces two types of wavelets, the extremal phase $\mathrm{D}(L)$ and the least asymmetric $\mathrm{LA}(L)$. The difference between them lies only in their phase functions, i.e., $\theta^{(G)}($.$) in the polar representation$

$$
G(f)=\left[\mathcal{G}^{(D)}(f)\right]^{1 / 2} e^{i \theta^{(G)}}(f)
$$


The Haar wavelet or $\mathrm{D}(2)$, is a filter of width $L=2$, that can be defined through its wavelet filter,

$$
h_{0}=\frac{1}{\sqrt{2}} \text { and } h_{1}=\frac{-1}{\sqrt{2}},
$$

or, equivalently, through its scaling filter,

$$
g_{0}=g_{1}=\frac{1}{\sqrt{2}} .
$$

The scaling coefficients for the LA(8) and other Daubechies wavelets are given in Daubechies (1992).

\section{Trend}

We describe the trend here as a long term change in the underlying mean level per unit time. For example, recent concerns about the possibility of climate change have focused attention on temperature series. A crucial question raised by these data is whether the temperature rise of around $0.5^{\circ} \mathrm{C}$ could be interpreted as a trend over the last hundred years, see Bloomfield and Nychka (1992). This trend can be a start of a systematic warming, or an effect of natural variability. The most difficult problem when testing for linear trend is the presence of dependence among the residuals. Because the residuals are dependent, the tests for trend based on the classical OLS regression will be inappropriate. A plausible statistical model for temperature series can consist of a mean level, a possible trend plus a noise term which can be a FD process. However, there are different models for describing various forms of trend, both linear and non-linear, and both stochastic and deterministic. In this work we will study only the model that represents the time series by using a pth-order polynomial function, that is

$$
T_{t}=\beta_{0}+\beta_{1} t+\beta_{2} t^{2}+\ldots+\beta_{p} t^{p}
$$

When $p=0$, there is no long-run increase or decline in the time series over time, and $p=1$, implies that there is a straight-line long-run growth (if $\beta_{1}$ $>0$ ) or decline (if $\beta_{1}<0$ ) over time. These simple linear trends are often called a deterministic linear trends. 


\subsection{Trend tests}

In this section we will provide three methods for testing the trend:

- the OLS test,

- the wavelet based test and,

- the band periodogram based test.

Yajima (1988) considered a polynomial regression consisting of a polynomial trend with $p=1$ and a stationary process with long memory. Based on that we consider the following model

$$
Z_{t}=T_{t}+X_{t}=a+\beta t+X_{t} \text { for } t=0, \ldots N-1
$$

where $X_{t}$ is a $\operatorname{FD}(d)$ process.

\subsection{Ordinary least squares method for testing the trend}

In this method we attempt to estimate the parameter $\beta$ by using the OLS method, i.e. by regressing $Z_{t}$ on $t$ in (14), and we consider the following two cases:

Case 1: $d=0$. That is, the $X_{t}$ is a zero mean white noise process of constant variance $\sigma_{X}^{2}$. It is well known that the least squares estimator of $\beta$ in this case is given by

$$
\hat{\beta}=\frac{\sum_{t=0}^{N-1}(t-\bar{t}) Z_{t}}{\sum_{t=0}^{N-1}(t-\bar{t})^{2}}
$$

with standard error

$$
s e(\hat{\beta})=\frac{\sigma_{X}^{2}}{\sqrt{\sum_{t=0}^{N-1}(t-\bar{t})^{2}}}
$$

In this case and under the null hypothesis $H_{0}$ that $\beta=0$ (against the alternative hypothesis $H_{1}$ that $\beta \neq 0$ ), we consider the following test statistic

$$
L S \equiv \frac{\hat{\beta}}{s e(\hat{\beta})},
$$


which approximately follows the $N(0,1)$ distribution.

Case 2: $0<d<\frac{1}{2}$. Yajima (1988) has shown that, for $0<d<\frac{1}{2}$, the estimator $\hat{\beta}$ is inefficient. By normalising the above estimate $\hat{\beta}$ by $N^{d}$, we get the same convergence as for Case 1, see Beran (1994) and Craigmile (2000). In Section 4 we will calculate corrected critical values for the LS test for different $d$ 's via a Monte Carlo method.

\subsection{A wavelet-based test for testing the trend}

We investigate here the wavelet-based approach for testing the trend. In section 2 we introduced the DWT coefficients and we have seen that these can be divided into two different types: scaling coefficients $V$ and wavelet coefficients $W$. Percival and Bruce (1998) and Craigmile (2000) have shown the ability of the Daubechies wavelet filters to identify trend contaminated FD processes due to of the inherent differencing operations in the filters. This property implies that a Daubechies wavelet filter can transform a FD process of order $L / 2$ or less into

- a stationary process, i.e., a process with a lower dependency than the original data series, or

- can annihilate a polynomial of order less than $L / 2$.

This result only applies to the non-boundary coefficients.

Thus we can rewrite (14) in terms of the inverse wavelet and scaling coefficients as

$$
Z=S_{J}+\sum_{j=1}^{J} D_{j},
$$

where $S_{J}$ represents the trend and $\sum_{j=1}^{J} D_{j}$ represents the residuals. The above form is valid only for the Haar wavelet since there are no boundary coefficients in the Haar wavelet. Craigmile (2000) has generalised (16) for $L>2$. If $W=\mathcal{W}_{B D} W+\mathcal{W}_{S} W+\mathcal{W}_{B I} W$, then we can write

$$
\begin{aligned}
Z & =\mathcal{W}^{T} W \\
& =\mathcal{W}^{T}\left(\mathcal{W}_{B D}+\mathcal{W}_{S}\right) W+\mathcal{W}^{T} \mathcal{W}_{B I} W
\end{aligned}
$$

10 


$$
\equiv \hat{T}+\hat{X}
$$

where $\mathcal{W}_{B D}$ is the non-boundary coefficients matrix, $\hat{T}$ captures the trend and $\hat{X}$ represents the residuals. For more details about the statistical properties of the trend estimate, see Craigmile (2000). The idea of the wavelet based test for trend is simple. We would like to test the null hypothesis

$$
H_{0}: T_{t}=0 \quad t=0, \ldots, N-1 .
$$

Craigmile (2000) presented a test statistics for discriminating between this null hypothesis and the alternative hypothesis

$$
H_{1}: T_{t} \neq 0 \text { for some } t=0, \ldots, N-1 \text {. }
$$

The test statistic is defined by

$$
W T=\frac{\|V\|^{2}+\left\|W_{B D}\right\|^{2}}{\left\|W_{B I}\right\|^{2}} .
$$

Almasri (2000) introduced a test statistic for discriminating between the null hypothesis (17) and the alternative hypothesis (18) in the same way as in (19), but by taking into account all the wavelet and scaling coefficients. An alternative test statistic introduced by Craigmile (2000) and is given by

$$
W T^{*}=\frac{\|Z-\bar{Z}\|^{2}}{\left\|W_{B I}\right\|^{2}} .
$$

In each case the null hypothesis is rejected for large values of the test statistics.

\subsection{A band-periodogram test for trend}

In this section we will describe tests which based on the periodogram to assess the significance of trend in the FD processes. Time series analysts often identify periodicities and trends in the spectral density of a time series. As we have seen in section 2, we can partition the periodogram into two parts, the high frequency part $\hat{S}^{H}($.) (identifies periodicities) and the low frequency part $\hat{S}^{L}($.$) (identifies trends). These two parts can help us build new tests for the$ trend. To illustrate the impact of the trend on the periodogram of the $\operatorname{FD}(d)$ process, Figure (1) shows a periodogram for two $\mathrm{FD}(d)$ processes with (boxes) 
and without linear trend (circles) and when $d=0$ and $d=0.4$, respectively. In each case we have a sample length of $N=64$. We note that the trend has the strongest effect on the low frequency part of the periodogram only. The idea of the tests is simple. We want to know whether the contribution of the low frequency part of the periodogram to the high frequency part is significant. Under the null (17) we expect that the contribution of the low frequency part to the high frequency part is negligible. Hence we propose the test statistic

$$
B T=\frac{\hat{S}^{L}(f)}{\hat{S}^{H}(f)}=\frac{\sum_{q=1}^{N / 2^{J}} \hat{S}\left(f_{q}\right)}{\sum_{j=1}^{J-1} \sum_{i=N / 2^{j+1}}^{m} \hat{S}\left(f_{i}\right)},
$$

where $N=2 m+1$ if $N$ odd and $N=2 m+2$ if $N$ even. We reject the null for large values of $B T$. In a similar manner as in Section 3.3, we propose a test to examine the existence of a trend. Define

$$
B T^{*}=\frac{\sum_{t=0}^{N-1}\left(Z_{t}-\bar{Z}\right)^{2}}{\sum_{j=1}^{J-1} \sum_{i=N / 2^{j+1}}^{m} \hat{S}\left(f_{i}\right)} .
$$

When $d=0$, the test statistic (21) has approximately an $F$ distribution since the numerator and the denominator are independent and follow approximately $\chi^{2}$ distributions.

\section{The size and the power of the tests}

This section describes the results of a Monte Carlo study designed to assess the size and the power properties of the tests described in the previous section. We calculate the corrected critical values for the $L S$ test for different $d\left(L S^{*}\right)$ by using a Monte Carlo simulation study. The critical values for the $B T, B T^{*}$ and $W T$ have also been obtained by simulation. Almasri (2000) obtaines the critical values for the $W T$ but by taking into account the boundary coefficients. For the $W T^{*}$ test we use the critical values that have been listed in Craigmile (2000, page 108). For the $L S^{*}, B T, B T^{*}$ and $W T$ tests we obtain the critical values by simulation. We simulate a 10000 of $\operatorname{FD}(d)$ processes with no trend, and calculated the resulting test statistic for the $L S^{*}, B T, B T^{*}$ and $W T$. The length of the series is 1024. Table 1 lists the upper quantiles of the distribution of the $L S^{*}, B T, B T^{*}$ and $W T$

12 
a
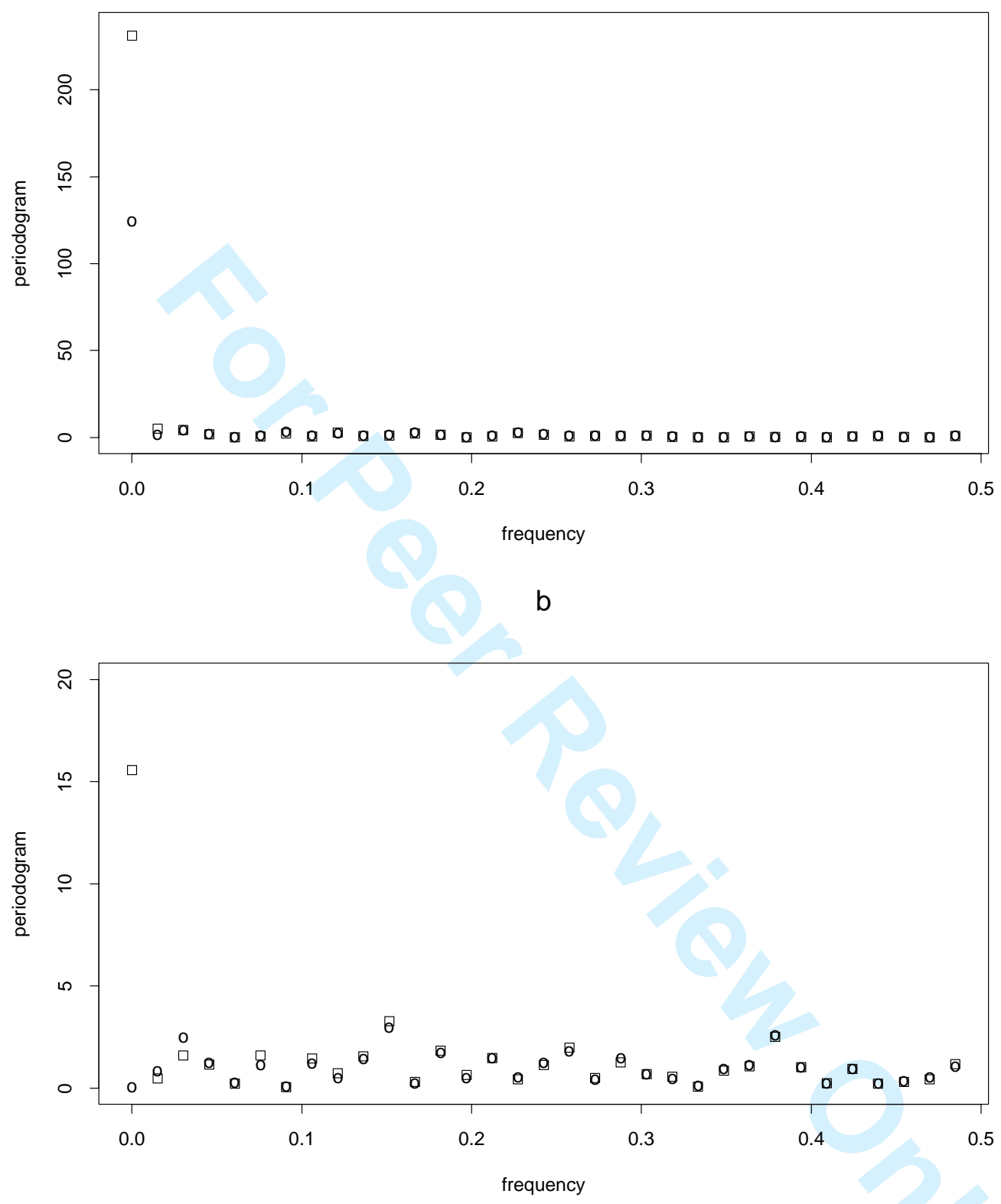

Figure 1: (a) The periodogram for a realisation when $d=0$, $\square$ with trend $(b=$ 1/64) and $\circ$ without trend. (b) The periodogram for a realisation when $d=0.4, \square$ with trend $(b=1 / 64)$ and $\circ$ without trend. 


\begin{tabular}{cc|ccc} 
test & $d$ & $\mathrm{p}=0.90$ & $\mathrm{p}=0.95$ & $\mathrm{p}=0.99$ \\
\hline$B T$ & 0 & 0.0109 & 0.0133 & 0.0178 \\
& 0.20 & 0.1076 & 0.1405 & 0.1961 \\
& 0.40 & 2.0942 & 2.8262 & 4.3252 \\
\hline$B T^{*}$ & 0 & 2.0176 & 2.0214 & 2.0304 \\
& 0.20 & 2.0975 & 2.1218 & 2.1648 \\
& 0.40 & 2.5310 & 2.6763 & 2.9604 \\
\hline$W T$ & 0 & 0.013 & 0.014 & 0.019 \\
& 0.20 & 0.087 & 0.105 & 0.136 \\
& 0.40 & 1.183 & 1.559 & 2.329 \\
\hline$L S^{*}$ & 0 & 1.282 & 1.645 & 2.326 \\
& 0.20 & 4.749 & 6.184 & 8.715 \\
& 0.40 & 25.621 & 32.852 & 46.202 \\
\hline
\end{tabular}

Table 1: Table of the 90th, 95th and 99th quantiles of the $B T, B T^{*}, W T$ and $L S^{*}$, tests for various values $d$. The evaluation of the distribution was based on 10000 simulations of a FD process using a DH algorithm.

for different $d$, where $J=8$ using Haar wavelet. As we can see from the table that the critical values of the tests increases with $d$.

\subsection{Empirical size}

Here we present our main results along with results of our Monte Carlo experiment regarding the dominating effects on the size of the above tests. We calculate the estimated size by observing how many times the null is rejected in repeated samples under conditions where the null is true, which called for type 1 error. We simulate $10000 \mathrm{FD}(d)$ processes with no trend (using different seed than the critical values simulation), and calculate how many times the null is rejected where the null is true. Table 2 gives the results of the size for the $L S^{*}, B T, B T^{*}$ and $W T$, where $J=8$ and by using Haar wavelet for the $W T$ test. The results have shown that the tests performs well as regarding the size when using the simulated critical values in Table 2. 


\begin{tabular}{cc|ccc} 
test & $d$ & $\mathrm{p}=0.10$ & $\mathrm{p}=0.05$ & $\mathrm{p}=0.01$ \\
\hline$B T$ & 0 & 0.101 & 0.052 & 0.014 \\
& 0.20 & 0.104 & 0.050 & 0.017 \\
& 0.40 & 0.094 & 0.049 & 0.012 \\
\hline$B T^{*}$ & 0 & 0.113 & 0.055 & 0.010 \\
& 0.20 & 0.112 & 0.053 & 0.015 \\
& 0.40 & 0.121 & 0.056 & 0.013 \\
\hline$W T$ & 0 & 0.109 & 0.067 & 0.012 \\
& 0.20 & 0.125 & 0.063 & 0.020 \\
& 0.40 & 0.100 & 0.053 & 0.014 \\
\hline$L S^{*}$ & 0 & 0.084 & 0.0516 & 0.011 \\
& 0.20 & 0.010 & 0.052 & 0.010 \\
& 0.40 & 0.097 & 0.049 & 0.011 \\
\hline
\end{tabular}

Table 2: Empirical sizes for $B T, B T^{*}, W T$ and $L S^{*}$.

\subsection{Empirical power}

In this sub-section we discuss the most interesting results of our Monte Carlo experiment, regarding the power of the above tests which means the rejection of the null when null is false. The power results are estimated by calculating rejection frequencies from 1000 replication for different trend parameters $(\beta)$, different $d$ and $N=1024$, where $J=8$ and by using LA(8) wavelet for the $W T^{*}$ and Haar wavelet for the $W T$, see Figure 2 and Figure 3 . We can see from Figure 2 that the power of the $W T$ (using LA(8) wavelet) and $B T$ is less than that of the $L S$ test, when $d=0$ and $d=0.2$. The $W T$ performs better than $L S$ and $B T$ when $d=0.4$ and $d=0.49$. However, the $B T$ performs reasonably well when $d=0.49$, but is quite a bit less than $W T$. Figure 3 shows that the $L S^{*}$ performs better than other tests for $d=0, d=0.20$ and $d=0.40$. We can also see that the $B T^{*}$ performs better than $W T^{*}$ (using Haar wavelet). In summary, the $L S^{*}$ and $L S$ tests perform better when the FD parameter is smaller, and the wavelet based tests and the band periodogram based tests are more better as $d$ approximate $\frac{1}{2}$. 

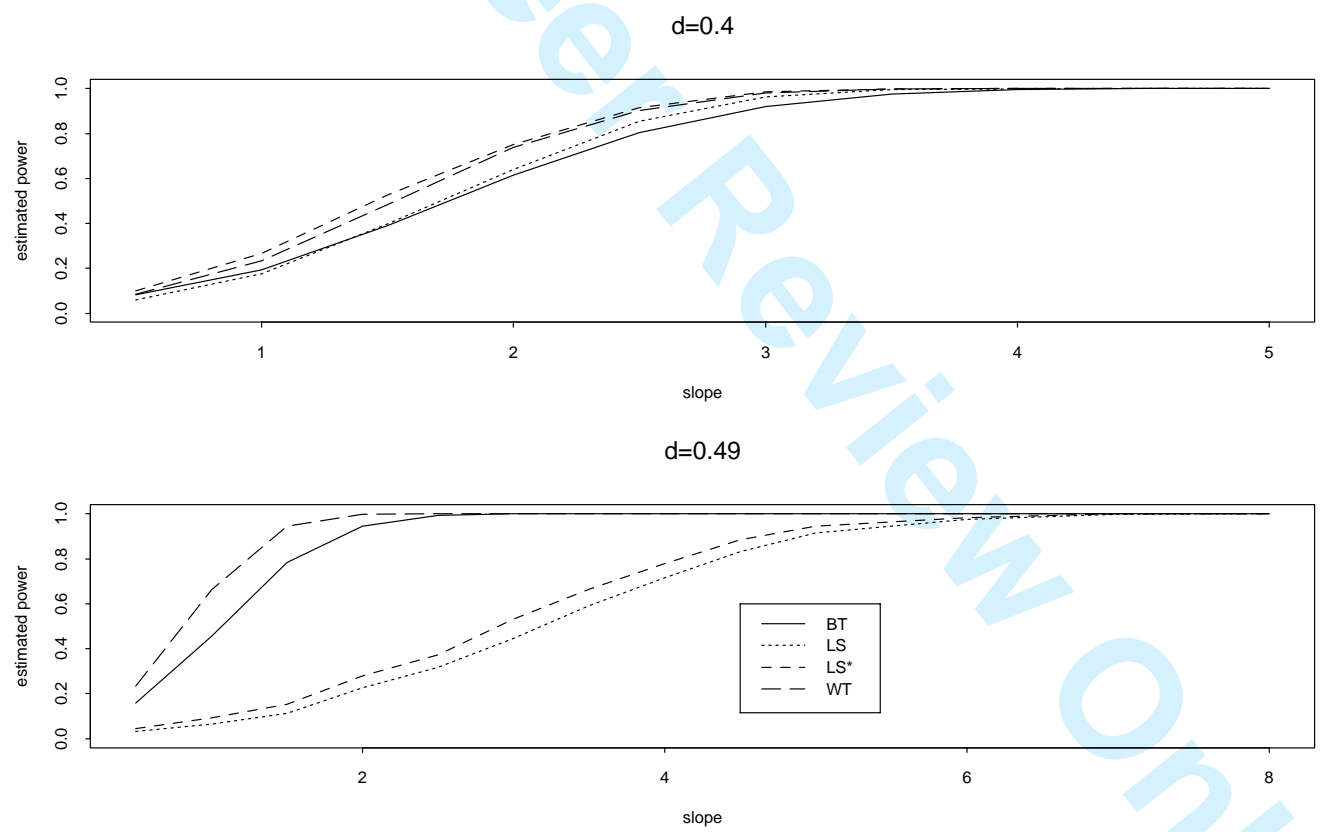

Figure 2: Figures show a comparison of the power of the test for trend for various slopes and a $d$. The sample size used was 1024, with a DWT using the LA(8) filter based on the Craigmile's critical values and analysing to $J=8$ levels. 
$d=0$
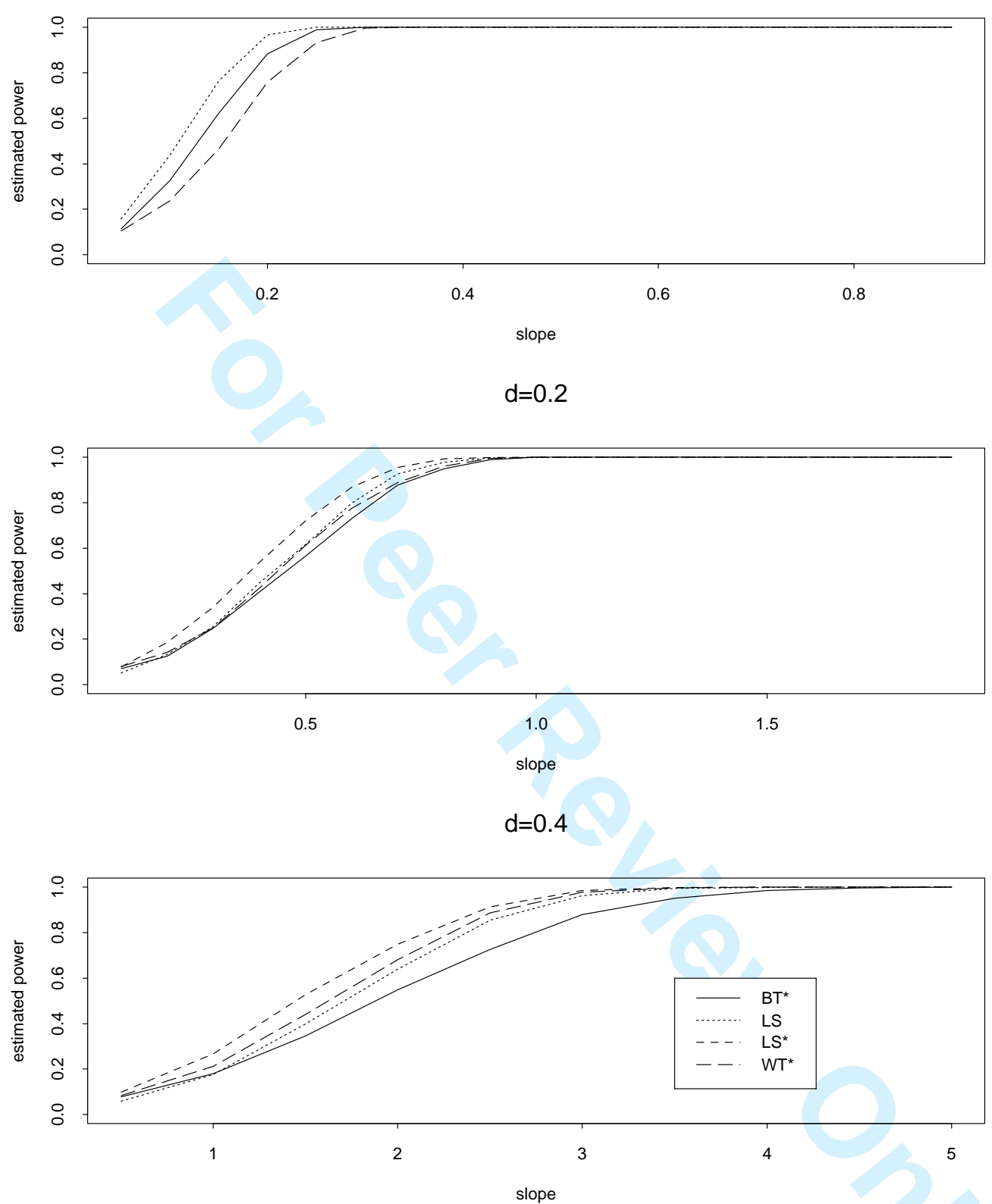

Figure 3: Figures show a comparison of the power of the test for trend for various slopes and a $d$. The sample size used was 1024, with a DWT using the Haar filter and analysing to $J=8$ levels.

17

URL: http://mc.manuscriptcentral.com/Issp E-mail: comstat@univmail.cis.mcmaster.ca 


\section{Summary}

In this paper we introduced two tests for testing the trend in LM processes, based on the wavelet and band periodogram. These tests have been compared with the OLS based test. The results have shown that the $L S^{*}$ and $L S$ tests perform better when the FD parameter is smaller, and the wavelet based tests and the band periodogram based tests are much better as $d$ approximate $\frac{1}{2}$.

\section{References}

Almasri, A. (2000) Some Aspects of Wavelet Analysis in Time Series. Research Report, Department of Statistics, Göteborg University, Sweden.

Beran, J. (1994) Statistics for Long-Memory Processes. New York: Chapman and Hall.

Bloomfield, P. and Nychka, D. (1992) Climate Spectra and Detecting Climate Change. Climatic Change, 21, 275-287.

Brillinger, D. R. (1994) Some River Wavelets. Environmetrics, 5,211-220.

Brillinger, D. R. (1996) Some Uses of Cumulants in Wavelet Analysis. Nonparametric Statistics, 6, 93-114.

Craigmile, P. F. (2000) Wavelet-Based Estimation for Trend Contaminated Long Memory Processes. Ph.D. dissertation, Department of Statistics, University of Washington, Seattle.

Daubechies, I. (1992) Ten Lectures on Wavelets. Philadelphia: SIAM.

Deo, R. S. and Hurvich, C. M. (1998) Linear Trend with Fractionally Integrated Errors. J. Time Ser. Anal. 19, 379-397.

Granger, C. W. J. and Joyeux, R. (1980) An Introduction to Long-Memory Time Series Models and Fractional Differencing. Journal of Time Series Analysis, 1, 15-29.

Hosking, J. R. M. (1981) Fractional Differencing. Biometrika, 68, 165-76.

Künsch, H. (1986) Discrimination between Monotonic Trends and LongRange Dependence. Journal of Applied Probability, 23, 1025-1030.

Mallat, S. (1989) A Theory for Multiresolution Signal Decomposition: The wavelet Representation. IEEE Transactions on Pattern Analysis and Ma-

18

URL: http://mc.manuscriptcentral.com/Issp E-mail: comstat@univmail.cis.mcmaster.ca 
chine Intelligence, 11, 674-93.

Percival, D. B. and Walden, A. T. (1993) Spectral Analysis for Physical Applications. Cambridge, UK: Cambridge University Press.

Percival, D. B. and Walden, A. T. (2000) Wavelet Methods for Time Series Analysis. Cambridge, UK: Cambridge University Press.

Priestley, M. B. (1981) Spectral Analysis and Time Series. London: Academic Press.

Schuster, A. (1898) On the Investigation of Hidden Periodicities with Application to a Supposed 26 Day Period of Meteorological Phenomena. Terrestrial Magnestism, 3, 13-41.

Smith, R. L. (1993) Long-Range Dependence and Global Warming. In Statistics for the Environment, pp. 141-161. Wiley (New York).

Yajima, Y. (1988) On Estimating of a Regression Model with Long-Memory Stationary Errors. Ann. Statist., 16, 791-807. 